\title{
Catalogue of the tick collection (Acari, lxodida) of the Museu de História Natural Capão da Imbuia, Curitiba, Paraná, Brazil
}

\author{
Márcia Arzua ${ }^{1}$, Valeria C. Onofrio ${ }^{2} \&$ Darci M. Barros-Battesti ${ }^{2}$ \\ ${ }^{1}$ Museu de História Natural Capão da Imbuia, Prefeitura Municipal de Curitiba. Rua Benedito Conceição 407, \\ 82010-080 Curitiba, Paraná, Brasil. E-mail: marzua@terra.com.br \\ ${ }^{2}$ Laboratório de Parasitologia, Instituto Butantan. Avenida Vital Brasil 1500, 05503-900 São Paulo, São Paulo, Brasil. \\ E-mail:valcastilho@butantan.gov.br; dbattesti@butantan.gov.br
}

\begin{abstract}
The tick collection of the Museu de Historia Natural Capão da Imbuia was first sorted in the end of the 80's, and a list of specimens was published in 1992. During the last ten years, the collection has grown substantially and became the depository collection for type-specimens. The collection is the most regional representative depository of ticks from the State of Paraná, and it is one of the four largest Brazilian collections in number of specimens. All material was revised, and the data were computerized and catalogued in cards. To date, the collection includes 504 vials, with 2,073 specimens, belonging to 25 species and six genera of the families Argasidae and Ixodidae. The ticks were collected in regional and state parks and in green areas from 60 municipalities distributed within eight Brazilian states. This catalogue corrects some of the records published in 1992 for the state of Paraná and records new host species for Amblyomma aureolatum (Pallas, 1772), A. cajennense (Fabricius, 1787), A. Iongirostre (Koch, 1844), A. tigrinum Koch, 1844, Ixodes aragaoi Fonseca, 1935, I. auritulus Neumann, 1904, I. fuscipes Koch, 1844 and I. paranaensis Barros-Battesti, Arzua, Pichorim \& Keirans, 2003. Amblyomma brasiliense Aragão, 1908, A. coelebs Neumann, 1899, A. nodosum Neumann, 1899 and I. fuscipes are recorded, for the first time, from the state of Paraná; A. Iongirostre from Rio Grande do Sul; I. fuscipes from Santa Catarina; I. schulzei Aragão \& Fonseca, 1951 from Rondônia; and A. rotundatum Koch, 1844 from Bahia. In addition Haemaphysalis juxtakochi Cooley, 1946 and I. fuscipes are recorded for the first time, infesting birds.
\end{abstract}

KEY WORDS. Argasidae, hosts, Ixodidae, regional ixodofauna.

RESUMO. Catálogo da coleção de carrapatos (Acari: Ixodida) do Museu de História Natural Capão da Imbuia, Curitiba, Paraná, Brasil. O acervo de carrapatos do Museu de História Natural Capão da Imbuia começou a ser organizado no final dos anos 80 , tendo sua primeira listagem publicada em 1992. Nos últimos dez anos a coleção cresceu substancialmente e passou a conter tipos. Sua importância está na representatividade da ixodofauna regional do Paraná e também por ser uma das quatro maiores coleções brasileiras em número de espécimes tombados. Todo o material foi revisado, informatizado e catalogado em fichas. Atualmente possui 504 lotes com 2.073 exemplares, pertencentes a 25 espécies, distribuídas em seis gêneros das famílias Argasidae e Ixodidae. Os carrapatos tombados foram coletados em parques regionais, nacionais e de áreas verdes de 60 municípios de oito estados brasileiros. Através deste catálogo, corrige-se registros apontados em 1992 para o Estado do Paraná e registra-se novos hospedeiros para Amblyomma aureolatum (Pallas, 1772), A. cajennense (Fabricius, 1787), A. Iongirostre (Koch, 1844), A. tigrinum Koch, 1844, Ixodes aragaoi Fonseca, 1935, I. auritulus Neumann, 1904, I. fuscipes Koch, 1844 e I. paranaensis Barros-Battesti, Arzua, Pichorim \& Keirans, 2003. Amblyomma brasiliense Aragão, 1908, A. coelebs Neumann, 1899, A. nodosum Neumann, 1899 e I. fuscipes são registrados pela primeira vez para o Paraná, A. longirostre para o Rio Grande do Sul, l. fuscipes para Santa Catarina, I. schulzei Aragão \& Fonseca, 1951 para Rondônia e A. rotundatum Koch, 1844 para o Estado da Bahia. Também são registradas em aves, pela primeira vez no Brasil, as espécies Haemaphysalis juxtakochi Cooley, 1946 e I. fuscipes.

PALAVRAS-CHAVES. Argasidae, hospedeiros, Ixodidae, ixodofauna regional.

In chronological order, the first tick collection in Brazil was established by Henrique de Beaurepaire Rohan Aragão, in the beginning of the 20th century. He contributed with 6,833 tick specimens in 1,532 vials, distributed in 11 genera, deposited in the collection of the Instituto Oswaldo Cruz (IOC), Rio de Janeiro, State of Rio de Janeiro. Nevertheless, this collection

Revista Brasileira de Zoologia 22 (3): 623-632, setembro 2005 
was closed in 1994. Continuing the work of Aragão, N.M. SERRA FREIRE et al. (unpublished data) took over the responsibility for the collection and created a new one, which in 2002 included 3,667 specimens in 615 vials.

During the 30's, Flavio Oliveira Ribeiro da Fonseca, initiated the mite collection of the Butantan Institute, São Paulo, State of São Paulo. In 1963, the collection already included approximately 80.000 specimens (BARROS-BATTESTI \& KNYSAK 1999), of which approximately $30 \%$ were ticks. This is one of the most important collections in Latin America, mainly due to the conservation state of its specimens and also for including type material, not only from Brazil but also from other countries worldwide. The collection of the Butantan Institute includes, to date, 49 Brazilian species, distributed within nine genera, as well as 48 foreign species of 10 genera, totaling 14,468 specimens excluding those immatures that are only identified at genera level. Flavio da Fonseca was curator of the collection from 1931-1963. Curatorial activities were resumed during the 70 's and the collection was under the responsibility of Nélida Margarida Lizaso, untill 1992. Since 1995, the mite collection has been under the responsibility of Darci Moraes BarrosBattesti, having been assigned the abbreviation IBSP.

The tick collection of the Museu de Historia Natural Capão da Imbuia, started to be organized in the end of the 80 's, receiving the abbreviation MHNCI, and includes specimens that were collected casually by the naturalists André Mayer and Rudolph Lange, during their zoological expeditions throughout the state of Paraná, during the first half of the $20^{\text {th }}$ century. Ornithologists and mast zoologists contributed with material collected during the 80's, which resulted in the first list published by BARRos \& BAGGIO (1992). Between 1989 and 1991 the collection was under the responsibility of D.M. BarrosBattesti, followed by M. Arzua. During the last 10 years the collection increased substantially with material from periodical samplings in the state of Paraná as well as with material donated by specialists from other states. Due to the fact that the collection included types, regional representatives and also for being one of the four largest Brazilian collections in number of specimens, it stands out beside others that were created during the last decade.

The Coleção Nacional de Carrapatos da Faculdade de Medicina Veterinária e Zootecnia da Universidade de São Paulo (CNC-FMVZ/USP) was created in the end of the 90's, and in few years has amounted to 2,437 specimens distributed within 34 species and six genera. Since the beginning, this collection has been under the responsibility of Marcelo Bahia Labruna. The importance of this collection is attributed mainly to the fact that it includes abundant, recently collected material from the states of São Paulo, Minas Gerais and Rondônia.

Due to the regional representatives as well as the number of specimens included, the aim of this study was to update, catalogue and present new locality and host records, making known the species deposited in the MHNCI collection.

\section{MATERIAL AND METHODS}

All material included in the collection was revised and each vial received a new printed label with species identification, number of specimens, host, locality, geographical coordinates, collection date and collector. In addition, each vial received a label with the collection number written in India ink. All information was compiled in Excell worksheets and, for security reasons, catalogue cards organized according to number and species were kept also.

The identification of adult ticks was based on identification keys (Mendez-Arocha \& Ortiz 1958, Aragão \& Fonseca 1961, Jones et al. 1972, GUIMARÃEs et al. 2001) and on the comparison of the type specimens and material from the IBSP collection. The identification of the immature stages of Ixodes ticks was based solely on the comparison with specimens reared under laboratory conditions and included in the collection of the IBSP and CNC-FMVZ/USP. The identification of the immature stages of the genus Amblyomma was confirmed by means of molecular sequencing. With the exception of Anocentor nitens (Neumann, 1897), the nomenclature followed that proposed by Horak et al. (2002).

The nomenclature for birds and mammals was based on the official list of the Instituto Brasileiro do Meio Ambiente e Recursos Naturais Renováveis - IBAma (2003) as well as on the list proposed by FonseCA et al. (1996).

Abbreviations used throughout the text: (BRM) Bosque Reinhard Maack, (PEL) Parque Estadual das Lauráceas, (MHNCI) Museu de História Natural Capão da Imbuia, (PEMG) Parque Estadual Mata dos Godoy, (PNS) Parque Nacional de Superagüi, (FEP) Floresta Estadual do Palmito, (PEVV) Parque Estadual Vila Velha, (ARIE) Área de Relevante Interesse Ecológico, (PEC) Parque Estadual de Campinhos, (PCPA) Parque Copesul de Proteção Ambiental.

\section{RESULTS}

The collection includes 504 vials containing 2,073 specimens belonging to 27 species and seven tick genera. These were collected in regional and national parks and in green areas of 60 municipalities distributed throughout eight Brazilian states. Geographical coordinates, hosts and species are listed below:

Geographical coordinates. Rondônia: Ouro Preto D'Oeste $\left(10^{\circ} 44^{\prime} 53^{\prime \prime} \mathrm{S}, 62^{\circ} 12^{\prime} 57^{\prime \prime} \mathrm{W}\right)$; Bahia: Cumuruxatiba $\left(17^{\circ} 06^{\prime} 38^{\prime \prime} \mathrm{S}\right.$, $\left.39^{\circ} 10^{\prime} 56^{\prime \prime} \mathrm{W}\right)$; Mato Grosso do Sul: Aquidauana $\left(20^{\circ} 28^{\prime} 16^{\prime \prime} \mathrm{S}\right.$, $\left.55^{\circ} 47^{\prime} 14^{\prime \prime} \mathrm{W}\right)$; Minas Gerais: Indianópolis (1902'19"S, 4755' $\left.01^{\prime \prime} \mathrm{W}\right)$, Nova Ponte $\left(19^{\circ} 08^{\prime} 08^{\prime \prime} \mathrm{S}, 47^{\circ} 40^{\prime} 58^{\prime \prime} \mathrm{W}\right)$; São Paulo: Botu-

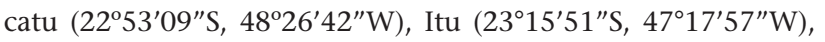

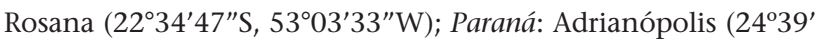
$26^{\prime \prime}$ S, 48 59'28"W), Adrianópolis - Parque Estadual das Lauráceas (PEL) $\left(24^{\circ} 44^{\prime} 11^{\prime \prime} \mathrm{S}, 48^{\circ} 32^{\prime} 53^{\prime \prime} \mathrm{W}\right)$, Almirante Tamandaré (25 $5^{\circ} 19^{\prime}$

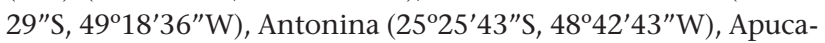
rana $\left(23^{\circ} 33^{\prime} 03^{\prime \prime} \mathrm{S}, 51^{\circ} 27^{\prime} 39^{\prime \prime} \mathrm{W}\right)$, Arapoti $\left(24^{\circ} 09^{\prime} 28^{\prime \prime} \mathrm{S}, 49^{\circ} 49^{\prime}\right.$ $\left.36^{\prime \prime} \mathrm{W}\right)$, Araucária $\left(25^{\circ} 35^{\prime} 35^{\prime \prime} \mathrm{S}, 49^{\circ} 24^{\prime} 37^{\prime \prime} \mathrm{W}\right)$, Bituruna $\left(26^{\circ} 09^{\prime}\right.$ 


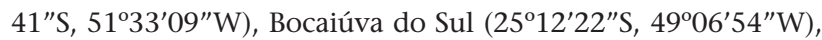

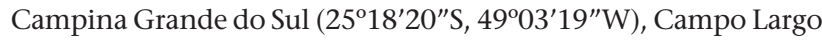

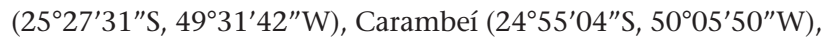

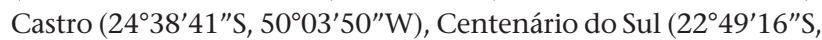
$\left.51^{\circ} 35^{\prime} 43^{\prime \prime} \mathrm{W}\right)$, Colombo ( $\left.25^{\circ} 17^{\prime} 30^{\prime \prime} \mathrm{S}, 49^{\circ} 13^{\prime} 27^{\prime \prime} \mathrm{W}\right)$, Curitiba $\left(25^{\circ} 25^{\prime} 40^{\prime \prime} \mathrm{S}, 4^{\circ} 16^{\prime} 23^{\prime \prime} \mathrm{W}\right)$, Fênix - Parque Estadual Vila Rica $\left(23^{\circ} 54^{\prime} 58^{\prime \prime} \mathrm{S}, 51^{\circ} 58^{\prime} 45^{\prime \prime} \mathrm{W}\right)$, Foz do Iguaçu $\left(25^{\circ} 32^{\prime} 52^{\prime \prime} \mathrm{S}\right.$, $\left.54^{\circ} 35^{\prime} 17^{\prime \prime} \mathrm{W}\right)$, Guarapuava $\left(25^{\circ} 23^{\prime} 43^{\prime \prime} \mathrm{S}, 5^{\circ} 27^{\prime} 29^{\prime \prime} \mathrm{W}\right)$, Guaraqueçaba $\left(25^{\circ} 18^{\prime} 24^{\prime \prime}\right.$ S, $\left.48^{\circ} 19^{\prime} 44^{\prime \prime} \mathrm{W}\right)$, Guaraqueçaba - Parque Nacional de Superagüi (PNS) $\left(25^{\circ} 11^{\prime} 49^{\prime \prime} \mathrm{S}, 48^{\circ} 21^{\prime} 51^{\prime \prime} \mathrm{W}\right)$, Guaratuba

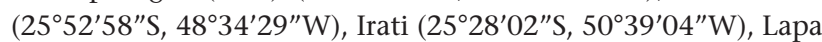

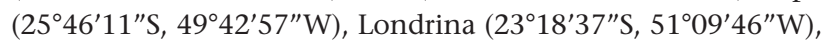
Londrina - Parque Estadual Mata dos Godoy (PEMG) (23 26'15"S, $\left.51^{\circ} 14^{\prime} 05^{\prime \prime} \mathrm{W}\right)$, Mandirituba $\left(25^{\circ} 46^{\prime} 44^{\prime \prime} \mathrm{S}, 49^{\circ} 19^{\prime} 34^{\prime \prime} \mathrm{W}\right)$, Mangueirinha $\left(25^{\circ} 56^{\prime} 28^{\prime \prime} \mathrm{S}, 52^{\circ} 10^{\prime} 32^{\prime \prime} \mathrm{W}\right)$, Maringá $\left(23^{\circ} 25^{\prime} 31^{\prime \prime} \mathrm{S}, 51^{\circ} 56^{\prime}\right.$

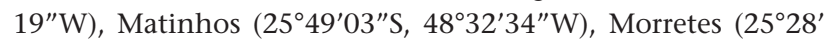

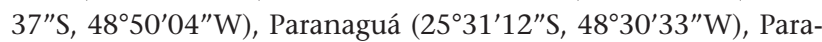
naguá - Floresta Estadual do Palmito (FEP) $\left(25^{\circ} 31^{\prime} 45^{\prime \prime} \mathrm{S}, 48^{\circ} 28^{\prime}\right.$

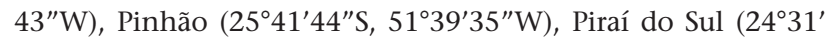
$34^{\prime \prime}$ S, 49 $\left.56^{\prime} 55^{\prime \prime} \mathrm{W}\right)$, Piraquara $\left(25^{\circ} 26^{\prime} 30^{\prime \prime} \mathrm{S}, 49^{\circ} 03^{\prime} 48^{\prime \prime} \mathrm{W}\right)$, Ponta Grossa $\left(25^{\circ} 05^{\prime} 42^{\prime \prime} \mathrm{S}, 50^{\circ} 09^{\prime} 43^{\prime \prime} \mathrm{W}\right)$, Ponta Grossa - Parque Estadual Vila Velha (PEVV) $\left(25^{\circ} 12^{\prime} 19^{\prime \prime} \mathrm{S}, 5^{\circ} 05^{\prime} 12^{\prime \prime} \mathrm{W}\right)$, Pontal do Paraná

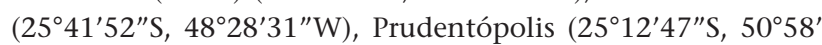

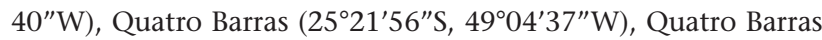

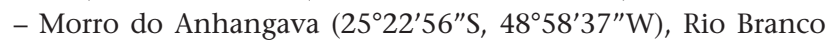
do Sul $\left(25^{\circ} 11^{\prime} 24^{\prime \prime} \mathrm{S}, 49^{\circ} 18^{\prime} 51^{\prime \prime} \mathrm{W}\right)$, Rio Ivaí - coordinates not found; Santo Antonio da Platina ( $23^{\circ} 17^{\prime} 42^{\prime \prime}$, 50 $\left.04^{\circ} 38^{\prime \prime W}\right)$, São José dos Pinhais $\left(25^{\circ} 32^{\prime} 05^{\prime \prime}\right.$ S, $\left.49^{\circ} 12^{\prime} 23^{\prime \prime} W\right)$, São Mateus do Sul ( $\left.25^{\circ} 52^{\prime} 27^{\prime \prime} \mathrm{S}, 50^{\circ} 22^{\prime} 58^{\prime \prime} \mathrm{W}\right)$, São Pedro do Iguaçu - Área de Rele-

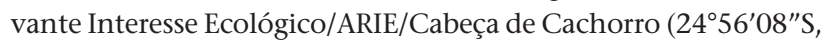
$\left.53^{\circ} 51^{\prime} 19^{\prime \prime} \mathrm{W}\right)$, Telêmaco Borba (24¹9'27"S, 50³6'58”W), Tijucas

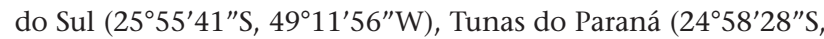
$\left.49^{\circ} 05^{\prime} 09^{\prime \prime} \mathrm{W}\right)$, Tunas do Paraná - Parque Estadual de Campinhos

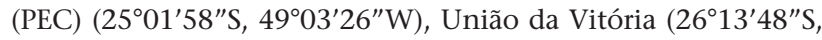

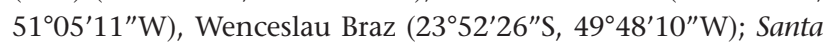
Catarina: Florianópolis $\left(27^{\circ} 35^{\prime} 48^{\prime \prime} \mathrm{S}, 48^{\circ} 32^{\prime} 57^{\prime \prime} \mathrm{W}\right)$, Ponte Alta do Norte $\left(27^{\circ} 09^{\prime} 30^{\prime \prime} \mathrm{S}, 50^{\circ} 27^{\prime} 52^{\prime \prime} \mathrm{W}\right)$, São Francisco do Sul $\left(26^{\circ} 14^{\prime}\right.$ 36"S, 48 38'17"W); Rio Grande do Sul: Triunfo - Parque Copesul

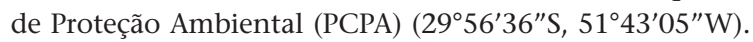

\section{Hosts}

Aves - Apodiformes: Apodidae-Streptoprocne biscutata (PL Sclater), Streptoprocne zonaris (Shaw); Columbiformes: Columbidae Columbina talpacoti Temminck; Coraciiformes: Momotidae Baryphthengus ruficapillus Vieillot; Galliformes: Cracidae Penelope obscura Temminck; Passeriformes: Conopophagidae - Conopophaga lineata (Wied-Neuwied), Dendrocolaptidae Campylorhamphus falcularius (Vieillot), Dendrocincla turdina (Lichtenstein), Sittasomus griseicapillus (Vieillot), Xiphorhynchus fuscus (Vieillot); Emberizidae - Basileuterus culicivorus (Lichtenstein), Basileuterus leucoblepharus (Vieillot), Euphonia pectoralis (Latham), Euphonia violacea (Linnaeus), Habia rubica (Vieillot), Haplospiza unicolor (Cabanis), Passerina brissonii (Lichtenstein), Pipraeidea melanonota (Vieillot), Poospiza lateralis (Nordmann), Pyrrhocoma ruficeps (Strickland), Saltator similis Lafresnaye \& d'Orbigny, Tachyphonus coronatus (Vieillot), Tangara seledon (Statius Müller), Tersina viridis (Illiger), Trichothraupis melanops (Vieillot), Zonotrichia capensis (Statius Müller), Formicariidae - Drymophila malura (Temminck), Mackenziaena severa (Lichtenstein), Thamnophilus caerulescens Vieillot, Thamnophilus ruficapillus Vieillot; Furnariidae - Cichlocolaptes leucophrus (Jardine and Selby), Clibanornis dendrocolaptoides (Pelzeln), Cranioleuca obsoleta (Reichenbach), Cranioleuca pallida (Wied), Furnarius rufus (Gmelin), Philydor atricapillus (Wied), Synallaxis cinerascens Temminck, Synallaxis ruficapilla Vieillot, Synallaxis spixi PL Sclater, Syndactyla rufosuperciliata (Lafresnaye), Muscicapidae - Turdus albicollis Vieillot, Turdus amaurochalinus Cabanis, Turdus nigriceps Cabanis, Turdus rufiventris Vieillot, Turdus subalaris (Seebohm); Pipridae - Chiroxiphia caudata (Shaw), Manacus manacus (Linnaeus); Troglodytidae - Troglodytes aedon Vieillot; Tyrannidae - Cnemotriccus fuscatus (Wied-Neuwied), Elaenia sp. Sundevall, Lathrotriccus euleri Cabanis, Mionectes rufiventris Cabanis, Myiarchus ferox (Gmelin), Myiodynastes maculatus (Statius Müller), Phylloscartes ventralis (Temminck), Tolmomyias sulphurescens (Spix); Pelecaniformes: Sulidae - Sula leucogaster (Boddaert); Piciformes: Picidae - Celeus flavescens (Gmelin); Strigiformes: Strigidae - Pulsatrix koeniswaldiana (Bertoni, M.\& Bertoni, W.).

Mammalia - Artiodactyla: Bovidae - Bos taurus L.; Cervidae Blastocerus dichotomus (Illiger), Mazama gouazoupira (G. Fischer), Mazama sp.; Tayassuidae - Tayassu pecari (L.); Carnivora: Canidae - Cachorro do mato, canídeo, Canis familiaris Linnaeus, Cerdocyon thous (L.), Crysocyon brachyurus (Illiger), Pseudalopex gymnocercus (Fischer); Felidae - Felis catus L., Herpailurus yagouaroundi (Lacépède), Puma concolor (L.); Mustelidae - Eira Barbara (L.), Galictis cuja (Molina), Lutra longicaudis (Olfers); Procyonidae - Nasua nasua (L.), Procyon cancrivorus (G. Cuvier); Chiroptera: Vespertilionidae-Eptesicus sp., Morcego; Didelphimorphia: Didelphidae - Didelphis albiventris Lund, Didelphis marsupialis L., Didelphis sp., Lutreolina crassicaudata (Desmarest), Monodelphis sp., Philander opossum (L.); Peryssodactyla: Equidae - Equus caballus L.; Tapiridae - Tapirus terrestris (L.); Primata: Cebidae - Cebus apella (L.); Hominidae - Homo sapiens (L.); Rodentia: Muridae - Rato silvestre; Erethizontidae - Sphiggurus sp., Sphiggurus spinosus (F. Cuvier), Sphiggurus villosus (F. Cuvier); Hydrochaeridae - Hydrochaeris hydrochaeris (L.); Xenarthra: Bradypodidae - Bradypus sp.; Myrmecophagidae - Myrmecophaga tridactyla (L.), Tamandua tetradactyla (L.).

Reptilia - Squamata, Serpentes: Viperidae - Bothrops leucurus Wagler.

\section{Species}

Amblyomma aureolatum (Pallas, 1772) - 182 vials (79 males, 69 females, 210 nymphs, 489 larvae).

Birds - B. leucoblepharus: 952 (1N, 1L), 22/X/1999; 963 (1N), 25/XI/1999; 966 (1N), 25/XI/1999; 969 (1N), 28/XII/1999;

Revista Brasileira de Zoologia 22 (3): 623-632, setembro 2005 
985 (1L), 28/IV/2000; 991 (1L), 29/V/2000; 993 (1L), 29/VI/ 2000, Curitiba, BRM; C. lineata: 935 (2N, 1L), 29/IX/1999, Curitiba, BRM; C. obsoleta: 907 (1N), 27/V/1999; 920 (3L), 28/VII/1999; 998 (7L), 29/V/2000; 1001 (1N, 2L) 28/VII/ 2000, Curitiba, BRM; C. pallida: 904 (1L), 27/V/1999; 905 (1L), 27/V/1999; 906 (2L), 27/V/1999, Curitiba, BRM; F. rufus: 853 (1N, 2L), 27/I/1999, Curitiba, BRM; P. lateralis: 953 (1N), 22/X/1999; 954 (2N), 22/X/1999, Curitiba, BRM; S. similis: 923 (1N, 1L), 27/VIII/1999; 974 (1N, 3L), 26/I/2000; 977(1L), 29/II/2000; 1002 (3L), 28/VII/2000, Curitiba, BRM; $S$. cinerascens: 1017 (4N, 1L), 31/X/2000, Curitiba, BRM; T. coronatus: 911 (1L), 28/VII/1999, Curitiba, BRM; T. caerulescens: 898 (2N, 37L), 24/III/1999; 979 (1L), 31/III/ 2000; 1000 (2N, 13L), 29/VI/2000; 1006 (1L), 30/VIII/2000, Curitiba, BRM; T. ruficapillus: 895 (21L), 29/IV/1999, Curitiba, BRM; T. aedon: 858 (1N), 26/II/1999; 896 (4N, 87L), 29/IV/1999; 897 (4N, 42L), 29/IV/1999; 899 (1N, 26L), 27/ V/1999; 965 (1N, 1L), 25/XI/1999; 984 (1N, 30L), 28/IV/ 2000; 1005 (2N), 30/VIII/2000, Curitiba, BRM; T. albicollis: 910 (1N), 27/V/1999; 913 (1N, 4L), 28/VII/1999; 917 (5L), 28/VII/1999, Curitiba, BRM; T. amaurochalinus: $946(1 \mathrm{~N})$, 22/X/1999; 956 (1N), 22/X/1999; 1018 (8N), 31/X/2000, Curitiba, BRM; T. rufiventris: $852(1 \mathrm{~N}) ; 854(1 \mathrm{~N}, 2 \mathrm{~L}) ; 855$ (2L); $856(1 \mathrm{~N}), 27 / \mathrm{I} / 1999 ; 857$ (1N), 26/II/1999; 894 (3L), 29/IV/1999; 900 (1N, 6L); 901(1L), 27/V/1999; 908 (36L), 29/IV/1999; 912 (1N); 914 (11L); 915-A (2N, 1L); 916 (1L); 918-A (3N, 2L); 919 (1L); 921 (2L); 922-A (1L), 28/VII/1999; 924 (1M, 14L); $925(2 \mathrm{~N}) ; 926(2 \mathrm{~N}) ; 927(1 \mathrm{~N}) ; 928(3 \mathrm{~N}) ; 929$ $(2 \mathrm{~N}) ; 930(1 \mathrm{~N}) ; 931(57 \mathrm{~L}) ; 932(6 \mathrm{~L}) ; 933(2 \mathrm{~N}, 1 \mathrm{~L}), 27 / \mathrm{VIII} /$ 1999; 934 (1N, 2L); $936(7 \mathrm{~N}) ; 937$ - A (1N); $938(1 \mathrm{~N}) ; 939$ $(3 \mathrm{~N}) ; 940(3 \mathrm{~N}) ; 941(3 \mathrm{~N}), 29 / \mathrm{IX} / 1999 ; 942(1 \mathrm{~N}) ; 944(1 \mathrm{~N})$; $945(2 \mathrm{~N}) ; 947(3 \mathrm{~L}) ; 948(3 \mathrm{~N}) ; 949(1 \mathrm{~N}) ; 950(3 \mathrm{~N}) ; 951(3 \mathrm{~N})$; $955(1 \mathrm{~N}), 22 / \mathrm{X} / 1999 ; 957(1 \mathrm{~N}) ; 958(1 \mathrm{~N}) ; 959(2 \mathrm{~N}) ; 960(3 \mathrm{~N})$; $961(1 \mathrm{~N}) ; 962(3 \mathrm{~N}) ; 964(1 \mathrm{~N}), 25 / \mathrm{XI} / 1999 ; 967(1 \mathrm{~N}) ; 968$ $(5 \mathrm{~N}) ; 970(1 \mathrm{~N}) ; 971(1 \mathrm{~N}) ; 972(3 \mathrm{~N}, 3 \mathrm{~L}) ; 973(1 \mathrm{~N}), 28 / \mathrm{XII} /$ 1999; 975 (2N), 26/I/2000; 978 (1L); 980 (1L), 31/III/2000; $981(2 \mathrm{~L}) ; 982(1 \mathrm{~N}) ; 983(1 \mathrm{~N}) ; 986(1 \mathrm{~N}) ; 987(1 \mathrm{~L}), 28 / \mathrm{IV} /$ 2000; $990(1 \mathrm{~N}), 29 / \mathrm{V} / 2000 ; 992$ (1L); 994 (1L); 996 (4N, 3L); 997 (1N); 999 (2N, 2L), 29/VI/2000; 1003 (3N), 28/VII/ 2000; 1007-A (1N, 1L), 30/VIII/2000; 1009 (1N); 1010 (1N); $1011(1 \mathrm{~N}) ; 1012(1 \mathrm{~L}) ; 1013(3 \mathrm{~N}) ; 1014(2 \mathrm{~N}, 1 \mathrm{~L}) ; 1015(2 \mathrm{~N}$, 1L); 1016 (2L), 29/IX/2000; 1019 (1N); $1020(4 \mathrm{~N}) ; 1021(2 \mathrm{~N})$; $1022(13 \mathrm{~N}) ; 1023(1 \mathrm{~N}), 31 / \mathrm{X} / 2000 ; 1024(1 \mathrm{~N}) ; 1025(2 \mathrm{~N})$; 1026 (19N, 3L); 1027-A (6N), 28/XI/2000; 1028 (1N, 1L); 1029 (4L); 1030 (1L); 1031 (6L), 27/XII/2000, Curitiba, BRM; Z. capensis: $1004(1 \mathrm{~N}), 30 / \mathrm{VIII} / 2000$, Curitiba, BRM.

Mammals - Unidentified wild dog: 146 (1F), 15/VI/1953, São Mateus do Sul; C. familiaris: 1376 (1F), 10/X/1992; 1520 (1F), 31/VII/1992, Almirante Tamandaré; 1399 (1F), 06/XII/1992, Antonina; 416 (1F), 11/XI/1998, Bocaiúva do Sul; 1378 (1F), 21/XII/1998; 1381 (1M), 26/I/2001; 1401 (1F), 25/III/2000, Campina Grande do Sul; 1369 (3M, 2F), 17/VI/2002, Colombo 422 (1F), 24/X/2001; 492 (1M, 1F), 14/XII/1999; 1522
(1F), 25/XI/1999; 988 (1F), 31/X/2001; 1361 (1F), 14/XII/ 1992; 1393 (1F), 04/I/2000; 1456 (2F), 14/X/2001; 1458 (1M, 1F), 31/X/2001; 1521 (1F), 30/IX/1996, Curitiba; 425 (1F),12/ II/2001, Guaraqueçaba; 1366 (13M); 1370 (5F),18/XII/1994, Mandirituba; 432-B (2M), 07/XI/1999; 1371(2F),15/III/2003; 1373 (2F), 13/III/2003, Piraquara; 1462 (1M, 1F),11/XI/2001, Rio Branco do Sul; 426 (1M), 03/IX/2001; 1460 (1M), 08/ IX/2001, Wenceslau Braz; C. thous: 1375 (2M, 1F), 09/V/ 2002, Araucária; 1369 (3M, 2F), 17/VI/2002, Colombo; 135 (3M), 19/V/1995; 160 (1M), 21/VII/1995, Curitiba; 107 (11M, 6F), 06/V/1993, Piraquara; 1239 (1F), 31/I/2000, São José dos Pinhais; F. catus: 1241 (1M), 13/IX/2001; 1374 (1F), 27/ X/2002; 1395 (1F), 07/X/1992, Curitiba; 1398 (2F), 07/XI/ 2000 Adrianópolis; H. sapiens: 1343 (1M), 30/VI/1993, São José dos Pinhais; 1350 (1M) 19/V/1995, Almirante Tamandaré; 1354 (1F), 28/II/2003, Curitiba; L. crassicaudata: 148 (1M), 1993, Ponta Grossa, PEVV; N. nasua: 1242 (1M), 09/ II/2000, Pinhão; P. cancrivorus: 113 (3M, 4F), 22/VIII/1993, Campina Grande do Sul; 423 (5M, 3F), 15/XI/2000, Prudentópolis; P. concolor: 118 (24M, 14F, 1N), 03/IX/1993, Curitiba; 173 (1M, 4F), 01/X/1995, Carambeí; T. tetradactyla: 1404 (1F), 18/II/2002, Telêmaco Borba.

Amblyomma brasiliense Aragão, 1908 - 3 vials (3 males, 2 females, 1 nymph).

Mammals - H. sapiens: 1353 (1M), 18/I/2000, Adrianópolis, PEL; T. terrestris: 50 (2M, 1F, 1N), 19/XI/1988, Foz do Iguaçu; 55B (1F), VIII/1945, Rio Ivaí.

Amblyomma cajennense (Fabricius, 1787) - 22 vials (31 males, 16 females, 39 nymphs, 55 larvae).

Birds - C. leucophrus: 1243 (42L), 30/VI/1990, Tijucas do Sul; C. lineata: 1246 (5L), 30/VI/1990, Tijucas do Sul; P. ruficeps: 1367 (3L), 30/VI/1990, Tijucas do Sul; T. coronatus: 1244 (4L), 30/VI/1990, Tijucas do Sul; T. albicollis: 1641 (1N), 04/ $\mathrm{X} / 2003$, Triunfo, PCPA.

Mammals - C. familiaris: 1640 (1M, 13N), 23/XI/2003, Rio Branco do Sul; E. caballus: 1438 (4M, 2F), 12/III/1992; 1439 (10M, 6F), 13/III/1992, Adrianópolis; H. sapiens: 6 (1F), 07/I/1987, Itu; 90 (2N), 25/X/1991, Pinhão; 99 (4M, 1F); 1342 (4M, 1F), 28/III/1992; 1524 (1N); 1525 (1N), 27/X/1993, Adrianópolis; 1574 (1M), 18/VII/1998, Aquidauana; 1345 (1F), 31/VII/1994, Guaraqueçaba; 430 (1M, 15N), 20/IX/1995, Indianópolis; $S$. villosus: 52-A (1F), 23/X/1990, Campina Grande do Sul; $T$. pecari: 119 - A (2M, 1F, 3N, 1L); 121 - A (1F, 3N); 122 - A (1M); 123 - A (3M, 1F), 16/IV/1994, Foz do Iguaçu.

Amblyomma calcaratum Neumann, 1899 - 7 vials (42 males, 16 females, 1 nymph).

Mammals - M. tridactyla: 1365 (35M, 9F, 1N), 28/XI/2002, Piraí do Sul; T. tetradactyla: 51 (1F), 23/II/1988, Paranaguá; 85 (1F); 89 (5M, 3F), 14/IX/1991, Arapoti; 1380 (1M); 1576 (1M, 1F), 28/VI/2000, Guarapuava.

Free in the environment: $1364(1 \mathrm{~F}), 25 / \mathrm{V} / 2002$, Curitiba. Amblyomma coelebs Neumann, 1899 - 1 vial (11 females). Mammals - T. terrestris: 55-A (11F), VIII/1945, Rio Ivaí. 
Amblyomma dubitatum Neumann, 1899 - 1 vial (23 males, 1 female, 2 larvae).

Mammals - H. hydrochaeris: 433 (23M, 1F, 2L), 15/I/1998, Curitiba.

Amblyomma geayi Neumann, 1899 - 9 vial (13 males e 4 females).

Mammals - Bradypus sp.: 15 (1M), date unknown, Curitiba Zoo; Sphiggurus sp.: 87-A (3M, 2F), 16/III/1992, Irati; 1397 (2M), 06/VI/1999, São José dos Pinhais; S. spinosus: 16 (1M), 07/ VI/1987, Mangueirinha; 31 (1M, 1F), 10/V/1990, Campina Grande do Sul; S. villosus: 48 (1M), 10/X/1990, Araucária; 7 (1M, 1F), 15/VII/1988, São José dos Pinhais; 39 (2M), date unknown; 1240 (1M), X/1989, Tunas do Paraná.

Amblyomma incisum Neumann, 1906 - 3 vials (4 males, 11 females, 2 nymphs).

Mammals - T. terrestris: 55-C (7F), VIII/1945, Rio Ivaí; 387 (3M, 4F), 17/III/1999, Adrianópolis, PEL.

Free in the environment: 1639 (1M, 2N), 05/XII/2003, Londrina, PEMG.

Amblyomma longirostre (Koch, 1844) - 54 vials (13 males, 10 females, 44 nymphs, 6 larvae).

Birds - B. ruficapillus: 1580 (1N), 06/V/1994, Fênix, Parque Estadual Vila Rica; B. culicivorus: $1252(1 \mathrm{~N}), 02 / \mathrm{V} / 2002$, Paranaguá, FEP; 1413 (1N), 28/VI/2002, São Pedro do Iguaçu, ARIE/Cabeça de Cachorro; C. flavescens: 1407 (1N), 13/I/ 1993, Piraquara; C. caudata: 1247 (2L), 27/III/2002; 1255 (1L), 02/V/2002, Paranaguá, FEP; C. lineata: 1538 (1N), 06/ II/1993, Piraquara; D. turdina: 1254 (1L); 1444 (1N), 02/V/ 2002, Paranaguá, FEP; E. pectoralis: 1405 (1N), 08/III/1995, Morretes; E. violacea: 1450 (1N), 19/XII/1990, Centenário do Sul; H. rubica: 1250 (1L), 27/III/2002, Paranaguá, FEP; L. euleri: 1651 (1N), 03/XI/2003, Triunfo, PCPA; M. severa: 41 (1N) Antonina 21/VIII/1990; M. rufiventris: 1249 (1L), 27/ III/2002, Paranaguá, FEP; M. manacus: 1422-A (1N), 15/V/ 2003, Paranaguá, FEP; M. ferox: 1568 (1N), 02/XI/1993, Tijucas do Sul; M. maculatus: 1530 (2N), 14/I/1993, Piraquara; P. brissonii: 1531 (1N), 18/X/1995, Nova Ponte; P. obscura: 1362 (2F), 31/VII/1991, Campina Grande do Sul; $P$. atricapillus: $1427(1 \mathrm{~N}), 08 / \mathrm{IV} / 2003$, Paranaguá, FEP; P. ventralis: $1618(1 \mathrm{~N}), 24 / \mathrm{XI} / 1993$, Guaraqueçaba, PNS; $P$. melanonota: $1650(1 \mathrm{~N}), 05 / \mathrm{XI} / 2003$, Triunfo, PCPA; $P$. koeniswaldiana: 1406-A (1N), 13/I/1993, Piraquara; S. similis: 1642 (2N); 1646 (1N), 04-XI-2003, Triunfo, PCPA; $S$. griseicapillus: $1638(1 \mathrm{~N}), 04 / \mathrm{XII} / 2003$, Londrina, PEMG; $S$. spixi: $1652(1 \mathrm{~N}), 03 / \mathrm{XI} / 2003$, Triunfo, PCPA; T. coronatus: 1621-A (1N), 23/XI/1993, Guaraqueçaba, PNS; 1645-B (1N), 04-XI-2003, Triunfo, PCPA; T. seledon: 1355 (1N), 16/VII/ 1990, Antonina; 1535 (1N), 14/VIII/1994, Morretes; T. viridis: 1356 (1N), 1993, Ponta Grossa; T. sulphurescens: 01 (2F), 17/ IV/1989, Morretes; 1493 (1N), 30/XII/1990, Antonina; T. melanops: 1408 (2N); 1409 (2N), 28/VI/2002, São Pedro do Iguaçu, ARIE - Cabeça de Cachorro; 1419 (2N); 1421-A (1N); 1426 (1N), 15/V/2003, Paranaguá, FEP; T. longirostris: 124
(1N), 27/III/2002, Paranaguá, FEP; T. amaurochalinus: 1648 (1N), 05/XI/2003, Triunfo, PCPA; T. rufiventris: 1467 (1N), 24/V/2000, São Pedro do Iguaçu, ARIE/Cabeça de Cachorro; 1502 (1N), 26/XII/1996, Centenário do Sul; T. subalaris: 1653 (1N), 05/XI/2003, Triunfo, PCPA.

Mammals - B. dichotomus: 419 (1F), 01-IX-1998, Rosana; $H$. sapiens: 436 (1N), 03/VII/1988, Fênix, Parque Estadual Vila Rica; Sphiggurus sp.: 86 (1M), 06/VII/1992, Almirante Tamandaré; 87-B (3M), 16/III/1992, Irati; 88 (1M, 2F), 16/ VI/1991, Araucária; 1577 (1M), 23/IX/1992, Pinhão; S. spinosus: 17(5M, 3F), 25/XI/1989, Arapoti; S. villosus: 49 (1M), 10/X/1990, Curitiba; 52-B (1M), 23/X/1990, Campina Grande do Sul.

Amblyomma nodosum Neumann, 1899 - 1 vial (1 male, 2 females).

Mammal -T. tetradactyla: 189 (1M, 2F), 03/VIII/1995, União da Vitória.

Amblyomma ovale Koch, 1844 - 28 vials (155 males, 97 females, 7 nymphs).

Mammals - B. dichotomus 1519 (1M), 01/IX/1998, Rosana; C. familiaris 384 (1F, $4 \mathrm{~N}), 14 / \mathrm{VII} / 1998$, Aquidauana; 1396 (1F), 12/III/1992; 1238 (2M, 2F), 13/III/1992, 1358 (2M), 16/III/ 1992, Adrianópolis; 1453 (1M); 1459 (2M, 2N), 2/XI/2001; 1372 (2F); 137 (2M), 03/XI/2001, Guaraqueçaba; 1637 (1M, 2F), 26/XII/2003, Morretes; 1368 (1F), 28/X/2002; 400 (1M, 3F),15/II/2003, São Francisco do Sul; C. apella 53 (1F), 19/ XI/1988, Foz do Iguaçu; C. thous 30 (5M, 1F), 14/V/1990, Piraí do Sul; E. barbara 1334 (37M, 24F), 08/XI/1998, Guaratuba; G. cuja 5 (1M, 1F), 29/XI/1986, Paranaguá; $H$. yagouaroundi 46 (1F), 30/XI/1989, Almirante Tamandaré; $H$. sapiens 1349 (1F), 11/II/1994, Morretes; L. longicaudis 20 (68M, 34F), 20/I/1990, Matinhos; N. nasua 13 (13N, 4F), 19/XI/1988, Foz do Iguaçu; P. cancrivorus 1387 (1M, 3F), 03/ IV/1992; 114 (1M, 1F), 18/V/1993, Adrianópolis; 83 (5M, 3F), 08/IV/1984, 82 (7M, 3F), 02/IV/1988, Matinhos; $P$. concolor 1391 (2M, 6F, 1N), 05/V/2002, Santo Antonio da Platina; S. villosus 52-C (1M), 23/X/1990, Campina Grande do Sul.

Free in the environment: 1442 (1M, 2F) 27/III/2002; 1443 (1M), 02/V/2002, Paranaguá, FEP.

Amblyomma rotundatum Koch, 1844 - 1 vial (2 females).

Reptile - B. leucurus 739 (2F), 22/XII/1998, Cumuruxatiba.

Amblyomma tigrinum Koch, 1844 - 3 vials (20 males, 6 females).

Mammals - C. brachyurus 108 (8M, 4F), 24/V/1993, Ponta Grossa; D. gymnocercus 76 (6M, 1F), VII/1983; 77 (6M, 1F), 16/IX/1983, Ponta Grossa, PEVV.

Amblyomma triste Koch, 1844 - 1 vial (1 male).

Mammal - Mazama sp. 435-B (1M), 10/V/2002, Santo Antonio da Platina.

Ixodes aragaoi Fonseca, 1935 - 1 vial (23 nymphs).

Mammal - Monodelphis sp.: 442 (23N), 08/XII/1999, Bituruna.

Ixodes auritulus Neumann, 1904 - 91 vials (57 females, 64 nymphs, 69 larvae). 
Birds - B. leucoblepharus: 592 (1L), 19/X/1994, Curitiba, Parque Barigüi; C. dendrocolaptoides: 609 (1F, 2N, 2L), 10/VIII/1995, Curitiba, Parque Passaúna; C. talpacoti: 580 (1F), 16/XI/1993, Curitiba, BRM; C. lineata: 102 (1N, 1L), 14/V/1992, Curitiba, Parque Passaúna; H. unicolor 1537 (1L), 08/II/1993, Piraquara; P. lateralis: 1610 (1N), 12/VII/1994, Curitiba, Parque Barigüi; 1605 (1N), 20/IV/1994, Curitiba, Parque Passaúna; S. ruficapilla: 909 (1N, 1L), 29/IV/1999, Curitiba, BRM; S. rufosuperciliata: 543 (1N), 25/III/1993; 573 (1N), 27/IX/1993, Curitiba, Parque Barigüi; 103 (1N), 14/V/1992; 557 (1N), 20/X/1993, Curitiba, Parque Passaúna; T. melanops: 604 (1N), 21/II/1995, Curitiba, Parque Barreirinha; T. albicollis: 91 (1F), 05/XII/1990; 1569 (1F), 17/I/1994; 1557 (1N),15/IX/1995, Curitiba, Parque Passaúna; T. amaurochalinus: 1595 (1N), 15/XII/1995, Almirante Tamandaré, Parque Primavera; 518 (2N), 23/III/1993, Curitiba, Parque Passaúna; T. nigriceps: 1593 (1L), 08/XII/1995, Curitiba, Parque Passaúna; 1597 (1F), 12/XII/1995, Curitiba, Parque Barreirinha; T. rufiventris: $606(1 \mathrm{~N}) ; 649(2 \mathrm{~N}, 4 \mathrm{~L}) ; 650(1 \mathrm{~N})$; 651 (1N), 08/IV/1995; 1606-A (1N); 1607-A (5N), 20/V/1995, Almirante Tamandaré, Parque Primavera; 570 (1N), 06/VII/ 1994; 581 (1F, 1N); 582 (1F); 583 (2F); 584 (1F), 16/XI/1993; 593 (1F); 594 (1F); 595 (1F), 20/X/1994; 599 (1F); 600 (1F); 601 (4F); $902(1 \mathrm{~N}) ; 903(1 \mathrm{~N}), 27 / \mathrm{V} / 1999 ; 915-\mathrm{B}(1 \mathrm{~L}) ; 918-\mathrm{B}$ (1N); 922-B (1L), 28/VII/1999; 937-B (1N), 29/IX/1999; 943 (2F), 22/X/1999; 995 (4L), 29/VI/2000; 1007-B (1N); 1008 (1N), 30/VIII/2000; 1027-B (2F), 28/XI/2000; 1570 (1F); 1571 (1F), 28/XII/1993; 1572 (3N, 13L); 1573 (1N, 4L), 06/VII/1994, Curitiba, BRM; 574 (1N); 575 (1F, 1N), 27/IX/1993; 576 (1L), 22/X/1993; 588-A (2N); 589 (2F); 590 (1F); 591(2F), 19/X/1994; 596 (1F); 597 (1F); 598 (1F), 18/XI/1994; 1611 (5L), 27/IV/ 1994; 605 (2N), 05/IV/1995; 608-B (8L), 29/VI/1995; 610 (1F), 04/X/1995 Curitiba, Parque Barreirinha; 572-A (1F, 1N), 23/ IX/1993, Curitiba, Parque Iguaçu; 92 (1F), 05/XII/1990; 93 (1N); 94 (2N, 4L), 14/V/1992; 100 (2N, 2L), 21/VI/1992; 101 (4F), 30/XI/1993; 558 (1L), 20/X/1993; 577 (1F, 1N); 578 (1F); $579(1 \mathrm{~N}), 10 / \mathrm{XI} / 1993 ; 585$ (1F); $586(2 \mathrm{~F}, 1 \mathrm{~N}) ; 587(1 \mathrm{~N}), 14 / \mathrm{X} /$ 1994; 603 (1N), 20/II/1995; 607-A (1N), 28/VI/1995; 611 (1F, 1N), 07/X/1995; 1492 (1F), 27/XII/1993; 1547 (11L); 1554 (1L), 16/V/1995; 1591 (1F); 1598 (1F); 1599 (1F), 08/XII/1995; 1603 A (5N, 2L); 1609 (2N), 29/VI/1994, Curitiba, Parque Passaúna; 1441 (6F), 28/X/1998, Quatro Barras.

Ixodes fuscipes Koch, 1844 - 11 vials (11 nymphs, 3 larvae).

Birds - B. culicivorus: 1526 (1N), 08/II/1992, Piraquara; C. falcularius: 1539 (1N), 06/II/1993, Piraquara; D. malura: 1553 (1N), 07/II/1993, Piraquara; S. rufosuperciliata: 1338-B (1N), 06/VIII/1991; 1543 (1N), 06/II/1993, Piraquara; T. coronatus: 1534 (1N), 07/II/1993, Piraquara; T. caerulescens: 1527 (1N), 06/II/1992, Piraquara; T. melanops: 1541-A (2N), 23/XII/ 1992, Adrianópolis; X. fuscus: 1578 (1N), 15/II/1993, Piraquara.

Mammals - L. crassicaudata: 418-A (1N),15/V/2000, Florianópolis; wild rodent: 1466-A (3L),18/VI/2001, Ponte Alta do Norte.
Ixodes loricatus Neumann, 1899 - 31 vials (20 males, 43 females, 4 nymphs, 3 larvae).

Mammals - D. albiventris: 420 (1F); 421 (1F), 20/XII/1983, Castro, Parque Estadual Caxambu; 125 (1M), 09/IV/1994; 144 - C (1M), 31/VII/1995; 149 - A (1M), 24/VIII/1995, Curitiba; 414 (1M, 1N), 08/XI/1999, Pinhão; 64 (1M); 66 (2M); 68-A (1M, 2F); 69-A (5M, 7F); 70 (1F); 71 - A (1M); 72 (2F), 18/IX/ 1983, Ponta Grossa, PEVV; D. marsupialis: 196 (1F), 09/V/ 1996, Curitiba; 61 (2M, 3F),I/XII/1983; 62-A (6F, 2L), 2/XII/ 1983, Ponta Grossa, PEVV; Didelphis sp.: 1392 (2F), 12/XI/ 2002, Campo Largo; 58 (1M, 1F), VIII/1945, Rio Ivaí; $L$. crassicaudata: 78-A (1F), 19/I/1984; 79 (1F); 80-A (1F); 81 (1F, 3N),18/IX/1983, Ponta Grossa, PEVV; N. nasua: 73 (1F), 10/IX/1983, Ponta Grossa, PEVV; P. opossum: 391 (2 F), 11/ XII/1991, Antonina; 57 (1F, 1L), 04/II/1945, Matinhos; 95 (1M, 3F), 05/II/1992; 1389 (1F), 03/II/1992, Pinhão; 1385 (1M, 1F), 25/XI/1996, Quatro Barras, Morro do Anhangava; 56 (1M, 1F), VIII/1945, Rio Ivaí; 360-A (1F), 06/VI/1996; 1237 (1F), 16/V/1996, Tunas do Paraná, PEC.

Ixodes paranaensis Barros-Battesti, Arzua, Pichorim \& Keirans, 2003 - 22 vials (1 male, 9 females, 17 nymphs, 2 larvae).

Birds - S. biscutata: Types (holotype, allotype and paratypes) 613 (1N), 11/VIII/1994; 614 (1N), 28/XI/1994; 615 (1F holotype), 30/XI/1994; $616(2 \mathrm{~F}, 3 \mathrm{~N}), 12 / \mathrm{XII} / 1994 ; 617$ (1F), 16/ XI/1994; 618 (1F), 09/XII/1994; 619 (1N), 25/XI/1994; 620 (1N), 30/XI/1994; $621(1 \mathrm{~F}), 20 / X I / 1994 ; 622(1 \mathrm{~N}), 12 / \mathrm{XII}$ 1994; 623 (1N), 30/XI/1994; 624 (1F), 28/XI/1994; 625 (2L), 15/IX/1995; 626 (1F), 23/X/1994; 629 (1M allotype), 18/VIII/ 1994; $630(1 \mathrm{~N}), 23 / \mathrm{II} / 1995 ; 631$ (1N), 19/I/1995; $632(1 \mathrm{~N})$, 09/XII/1994; 633 (1N), 23/II/1995, Morro do Anhangava, Quatro Barras. Additional material - 1363 (1F), 24/XI/1993, Quatro Barras, Morro do Anhangava, free in the environment; 1474 (2N), 08/IV/2000; 1475 (2N), 08/IV/2000, Ponta Grossa, Furnas I, PEVV; S. zonaris:, Ponta Grossa, PEVV.

Ixodes schulzei Aragão \& Fonseca, 1951 - 1 vial (1 female).

Free in the environment: $1523(1 \mathrm{~F}), \mathrm{X}-1980$, Ouro Preto D'Oeste. Anocentor nitens (Neumann, 1897) - 3 vials (13 males, 28 females, 2 nymphs).

Mammals - E. caballus: 1384 (9M, 17F), 30/III/1992, Adrianópolis; 424 (4M, 11F, 1N), 27/IX/2003, Rio Branco do Sul; C. familiaris: 432-A (1N), 07/XI/1999, Piraquara.

Rhipicephalus (Boophilus) microplus (Canestrini, 1888) - 13 vials (13 males, 73 females, 11 nymphs).

Mammals - B. taurus: 1463 (1F), 22/IX/2001, Botucatu; 541 (2F), 31/X/2001, Campo Largo; 1464 (2F), 20/XI/2001, Colombo; 394 (18F), IX-2003; 989 (6M, 6F), 07/XI/2001; 1454 (1M, 7F), 02/XI/2001; 493 (2F), 01/XI/2001, Guaraqueçaba; 1461 (1F), 20/XI/2001, Morretes; Canidae: 60 (1F), VII/1951, Curitiba; C. familiaris: 1457 (1F), 11/XI/2001, Rio Branco do Sul; Mazama sp.: 434 (3M, 10F, 10N); 435-A (3M, 22F, $1 \mathrm{~N}), 10 / \mathrm{V} / 2002$, Santo Antonio da Platina.

Rhipicephalus sanguineus (Latreille, 1806) - 6 vials (16 males, 15 females, 1 nymph, 1 larva). 
Mammals - C. familiaris: 1455 (1M), 20/X/2001, Curitiba; 1654 (2F), 04/XII/2003, Londrina; 1402 (2F), 14/II/2002, Maringá; 1357 (1M, 2F), 23/IV/1997; 1360 (1F),11/X/1992; 1379 (14M, $8 \mathrm{~F}, 1 \mathrm{~N}, 1 \mathrm{~L}), 03 / \mathrm{XI} / 2002$, Pontal do Paraná, Balneário Shangrilá.

Haemaphysalis juxtakochi Cooley, 1946 - 08 vials (6 males, 10 females, 13 nymphs, 3 larvae).

Birds - P. ruficeps: 1367 (3F), 30/VI/1990, Tijucas do Sul.

Mamíferos - H. sapiens: 1390 (1M); 05/XI/1992; 1394 (1F), 15/ XI/1996, Curitiba; M. gouazoupira: 1236 (3M, 2F, 12N, 1L), 19/V/2002, Prudentópolis; 59 (1F), VIII/1945, Vale do Ivaí; Mazama sp.: 1388 (1M, 2L), 07/VIII/1996, Guaratuba; 1386 (1M, 1F, 1N), 07/VIII/1996, Lapa; 1400 (2F), 06/V/1993, Pinhão.

Argas miniatus Koch, 1844 - 1 vial (50 females).

Free in the hen house: 1655 (50 F), Apucarana.

\section{DISCUSSION}

The tick species $A$. aureolatum was recorded for the first time in the state of Paraná by Guimarães (1945), from Caiobá in the municipality of Matinhos, infesting C. thous. RiвeIro (1966/ 1967 and 1970/1971) recorded this species from 25 municipalities, infesting domestic dogs and other mammals. Nevertheless, the depository of this material remains unknown. BARROs \& BAGGIO (1992) recorded the host species C. thous and G. cuja. However, these records are not considered valid because the $C$. thous specimens are in fact $A$. ovale and the G. cuja specimen is lost. The IBSP collection includes eight lots from the municipalities of Balsa Nova, Curitiba, Mallet, Piraí do Sul, Ponta Grossa, Reserva do Iguaçu and Wenceslau Braz, with specimens collected on dogs, cats and marsupials. All the immature specimens of this species were recently recorded on birds by ArzuA et al. (2003) in the municipality of Curitiba. Lutreolina crassicaudata can be considered a new host record for A. aureolatum.

All vials containing $A$. brasiliense and $A$. coelebs represent the first records of these species for the state of Paraná. While revising the material of $A$. naponense (Packard, 1869) and $A$. incisum, previously cited by BARROS \& BAGGIO (1992), we verified that the material belongs, in fact, to three different species. The one identified as $A$. naponense included a female of $A$. brasiliense, 11 females of $A$. coelebs and one female of $A$. incisum. On the other hand, the one identified as A. incisum is in fact, $A$. brasiliense. The species $A$. incisum was recorded from Ilha do Mel, in the municipality of Paranaguá, by Ribeiro (1966/1967), infesting a lizard. Nevertheless, this is considered a doubtfull record since this species is usually found infesting mammals, especialy tapir (GUIMARÃEs et al. 2001) and can eventually infest humans (Jones et al. 1972).

Amblyomma cajenense was previously recorded from eight municipalities in the state, infesting domestic dogs, capybaras, horses and humans (ARAGão 1936, RibEIRo 1966/1967, 1970/ 1971, SinKoc et al. 1998, GUIMARÃEs et al. 2001). In the IBSP col- lection there are only two vials from the state of Paraná: one, with no host record, from São José collected in 1937 (IBSP 1089 with two males), and the other collected on $C$. thous, from Japurá, in 1957 (IBSP 4599 with 1 male). The hosts Cichlocolaptes leucophrus and $P$. ruficeps are considered new records for the $A$. cajennense larval stages, since the other birds have already been recorded as hosts of the immature stages of this tick species in the state of Minas Gerais (RojAs et al. 1999).

Amblyomma calcaratum was recorded from Paraná by GUIMARÃEs et al. (2001), however, the authors did not comment on the collecting localities or hosts. On the other hand, a female identified as A. rotundatum by BARRos \& BAGGIO (1992), collected on T. tretradactyla from Paranaguá, is in fact $A$. calcaratum.

Despite being frequent on capybaras, there are few records of $A$. dubitatum from the state of Paraná, having been recorded only from Curitiba (ARAgão 1936) and Foz do Iguaçu (SINKOC et al. 1998). After 62 years, the specimens deposited in the MHNCI represent the second record of this species from Curitiba.

Amblyomma geayi was recorded from the municipalities of Mangueirinha, Araucária and Curitiba by BARros \& BAGGIO (1992) on S. villosus. Although there is one A. geayi specimen collected on Bradypus sp., deposited in the MHNCI collection, the host was donated to the local zoo without indication of its origin. It is certainly not from the state of Paraná, since the Bradypus distribution range does not reach southern Brazil.

The adult stages of $A$. longirostre are commonly found on rodents of the genus Sphiggurus, which is also a host for $A$. geayi. In addition to the specimens collected on Sphiggurus, the collection of the MHNCI also includes a female collected on $B$. dichotomus from Rosana, state of São Paulo and a nymph collected on a human being from Fênix, state of Paraná. The specimens collected on a Cervidae, although from the state of São Paulo, are probably the first record for this host. Amongst the material deposited in the IBSP collection there is also a female (IBSP 1278) collected on a human from São Paulo municipality as well as a vial with two males (IBSP 4974) collected on Sphiggurus from Paulo Frontin, state of Paraná. Correcting the records of BARROS \& BAGGIO (1992), vial 7 is lost and vial 14 is not $A$. longirostre, but could not be identified due to the fact that it is a nymph that is certainly not $A$. longirostre. Specimens from vial 31 belong to $A$. geayi and vial 52 includes one female A. cajennense, one male $A$. ovale and one male $A$. longirostre. In relation to the immature ticks, all vials deposited in the MHNCI collection include specimens collected on birds, with the exception of seven vials from Triunfo, RS and one vial from Nova Ponte, MG. All the remaining lots are from the state of Paraná. Aragão (1936) recorded birds of the genera Penelope, Saltator and Turdus as hosts of immature stages. Nevertheless, the author did not record the occurrence localities of these hosts. Thus, the bird species of the present study are considered new host records for A. longirostre, and the state of Rio Grande do Sul is considered a new locality record. 
The only vial containing $A$. nodosum, deposited in the collection of the MHNCI, was collected in União da Vitória and represents the first record of this species for the state of Paraná.

For A. ovale, all the locality and host records mentioned in the present study have been previously cited (ARAGÃo 1936, Ribeiro 1966/1967, 1970/1971, Barros \& Baggio 1992, SinKoc et al. 1998, Labruna et al. 2001, Guglielmone et al. 2003). The collection of the MHNCI also includes specimens collected on domestic dogs from the states of Mato Grosso do Sul and Santa Catarina, as well as one male collected on B. dichotomus, from Rosana, SP. Amongst the material deposited in the IBSP collection, there are two vials from the state of Paraná, with specimens collected on dogs from Wenceslau Braz (IBSP 831, 2 males) and Cruzeiro do Iguaçu (IBSP 6912, 3 females).

The two A. rotundatum females, deposited in the collection of the MHNCI, are from the state of Bahia and represent the first record for this species in the state. Despite having been recorded in the states of Santa Catarina and Rio Grande do Sul, by Aragão (1936) and Evans et al. (2000), the state of Paraná has not yet been included in the distribution range of this tick species, since the previous record, presented by BARros \& BAGGIO (1992), was mistaken.

Both $A$. tigrinum and $A$. triste have been previously recorded from the state of Paraná. The species A. tigrinum was collected on domestic dogs in the municipalities of Balsa Nova, Francisco Beltrão, Jacarezinho, Palmas, Palmeira and Porto Amazonas (Ribeiro 1970/1971). It was also recorded on $P$. gymnocercus, from Ponta Grossa (BARros \& BAGGIO 1992) and on B. dichotomus, from Foz do Iguaçu (Sinkoc et al. 1998). Thus, C. brachyurus represents a new host record for this species. Regarding A. triste it was recorded on $H$. hydrochaeris, from Foz do Iguaçu. Thus, the record from Santo Antonio da Platina is the second locality record for this species in the state of Paraná.

Although there is no A. oblongoguttatum Koch, 1844 material deposited in the MHNCI collection, this species was only recorded from Paraná by Ribeiro (1966/1967) on capybaras from the municipality of Campo Mourão. Curiously, a male, deposited in the IBSP collection, was recently found on a human from the Parque Estadual Mata dos Godoy, municipality of Londrina, $100 \mathrm{~km}$ from Campo Mourão. This record confirms the presence of this species in Paraná after almost 40 years. This tick species is known to occur in southeastern, centralwestern and northern Brazil, infesting domestic dogs, deer, tapir, wild pigs, bats and humans, according to the material deposited in the collections of the IBSP and CNC-FMVZ/USP and in the literature (Aragão 1936, Guerra \& Serra-Freire 1999, LABRUNA et al. 2000).

The occurrence of A. pseudoconcolor Aragão, 1908 in Southern Brazil was mentioned solely by GUIMARÃES et al. (2001), for the states of Paraná and Rio Grande do Sul, based on material deposited in the collection of the IBSP in 1957. The vial from Paraná includes a female collected on T. tetradactyla from
Jaguariaíva, PR and the one from Rio Grande do Sul (67 females and 2 nymphs) was collected on a Nothura maculosa (Temminck) bird from Viamão. Aragão (1936) recorded $A$. pseudoconcolor from the state of São Paulo, in the municipalities of Avaré, Faxina and Rio Pardo. The IBSP collection includes material from Botucatu, collected on armadillo in 1953. All the remaining material is from Maranhão, Bahia and Mato Grosso and was collected on armadillos and ant-eaters. Considering that the material deposited in this collection dates from the 1950's, the lack of recent records of this species in the South and Southeast is probably due to the lack of studies on Edentata in these regions.

Of the eight Ixodes species that occur in Brazil, four have been previously recorded in Paraná: I. aragaoi (RIBEIRo 1966/ 1967), I. loricatus (Guimarães 1945, Ribeiro 1966/1967, Barros \& BAgGio 1992), I. auritulus (Arzua et al. 1994, Arzua \& BarrosBattesti 1999, Arzua et al. 2003) and I. paranaensis (BARros-BATTESTI et al. 2003).

The species I. aragaoi is endemic for Brazil, with representative species only in the IBSP and CNC-FMVZ/USP collections. The adult stages were collected on Cervidae, unidentified wild dogs, horses, pumas and rodents. The IBSP collection includes the types from the municipality of Cotia, as well as material from other localities of the state of São Paulo, such as: Itapecerica da Serra, Embu-Guaçu, Lins and São Paulo. This collection also includes material from Foz do Iguaçu, PR and Pinheiro Machado, RS (BARRos-BATTESTI \& KNYSAK 1999). The CNCFMVZ/USP collection includes specimens from the states of Mato Grosso and São Paulo. Ribeiro (1966/1967) registered the first occurrence of this species in the state of Paraná, in the municipality of Pitanga. The material, collected on Monodelphis from Bituruna, deposited in the MHNCI collection, increases the number of hosts and the distribution range for I. aragaoi.

Three new host species are presented for $I$. auritulus, a species associated to birds: $H$. unicolor, $P$. lateralis e $T$. nigriceps. The municipalities of Piraquara and Quatro Barras are added to the species distribution range in the state of Paraná.

The species $I$. fuscipes is restricted to Panamá, Perú and Brazil. Previous records for the Brazilian territory include the states of São Paulo, Rio de Janeiro, Pará, Rondônia and Acre (Aragão 1936, Barros-Battesti \& Knysak 1999, Onofrio 2003). All material deposited in the MHNCI collection represents new host records for $I$. fuscipes, recorded for the first time on birds. In addition, the setentrional limits area stretched to the state of Santa Catarina.

The best represented Ixodes species in Brazilian collections is I. loricatus. It was recorded for the first time in the state of Paraná by Guimarães (1945), on P. opossum from Caiobá, Matinhos. Later, the species was recorded on domestic dogs from Antonina, on Didelphis sp. from Curitiba (RiberRo 1966/ 1967 ) and on D. marsupialis, D. albiventris, L. crassicaudata and N. nasua from Ponta Grossa (PEVV) (BARRos \& BAGGIO 1992). Balsa Nova and Pinhão were included in the species' distribu- 
tion range based on IBSP material. In this study, we add four new localities from the state of Paraná.

Known only by the type specimen, I. paranaensis was described based on specimens collected on S. biscutata from the Morro do Anhangava, Quatro Barras (BARRos-BAtTesti et al. 2003). The female and nymph paratypes (MHNCI 612) were deposited in the United States National Tick Collection, Georgia Southern University, Statesboro, USA; MHNCI 627 and MHNCI 628, a female and a nymph, respectively, were deposited in the IBSP collection; and MHNCI 630 (one nymph) was deposited in the Acari collection of the Zoological Museum of Moscow, Russia. The new records from the MHNCI collection increase the distribution range and add $S$. zonaris to the list of possible hosts for this species.

With less than 20 specimens deposited in the CNC-FMVZ/ USP, USNTC and IOC collections, I. schulzei was known only from the Southern (state of Santa Catarina) and Southeastern (States of São Paulo, Rio de Janeiro and Minas Gerais) regions (LABRUNA et al. 2003), infesting Nectomys squamipes (Brants). Although this species is not part of the Ixodes fauna of the state of Paraná, the female of $I$. schulzei, deposited in the MHNCI collection, is the first record of this species for the Northern region of the country.

Anocentor nitens, R. (B.) microplus, A. miniatus e R. sanguineus are common on domestic animals, occurring accidentally on wild animals. All of them have been previously recorded from the state of Paraná (Aragão 1936, FalCE et al. 1983, Ribeiro 1966/ 1967, Barros \& Baggio 1992, Labruna et al. 2001).

The occurrence of $H$. juxtakochi, in the state of Paraná, was recorded by Ribeiro (1966/1967) and BARRos \& BAGGIO (1992) on mammals of the genera Mazama and Tapirus. This species, very common on cervidae, was also found infesting wild rodents, tapir, quati and unidentified wild pigs (FAIRCHILD et al.1966, Jones et al.1972), as well as dogs, cattle and horses (Guglielmone et al. 1992). Beldomenico et al. (2003) found immature specimens on birds of the families Corvidae (Cyanocorax chrysops (Vieillot)) and Emberizidae (Arremon flavirostris Swainson), from Argentina. In this study we record the parasitism of $H$. juxtakochi on birds in Brazil. In addition we increase the species' distribution range in the state.

The MHNCI collection also includes 300 vials, with approximately 1,500 specimens, with immature ticks of the genera Amblyomma, Ixodes and Ornithodoros, from the states of Paraná, Minas Gerais, Mato Grosso do Sul, Rio Grande do Sul, Goiás, Distrito Federal and Sergipe. Approximately 1,300 specimens were collected on birds and the rest on mammals. The identification of these specimens will be carried out by means of molecular sequencing in the near future.

\section{ACKNOWLEDGMENTS}

To Marcelo Bahia Labruna, who kindly provided us compiled data from material deposited in the CNC-FMVZ/USP.

\section{REFERENCES}

Aragão, H.B. 1936. Ixodidas brasileiros e de alguns países limítrofes. Memórias do Instituto Oswaldo Cruz, Rio de Janeiro, 31 (4): 759-843.

Aragão, H.B. \& F. Fonseca. 1961. Notas de Ixodologia. VII. Lista e chave para os representantes da fauna ixodológica brasileira. Memórias do Instituto Oswaldo Cruz, Rio de Janeiro, 59 (2): 115-149.

Arzua, M.; D.M. Barros; P.M. Linardi \& J.R. Botelho. 1994. Noteworthy records of Ixodes auritulus Neumann, 1904 (Acari: Ixodida) on birds from Paraná, Southern Brazil. Memórias do Instituto Oswaldo Cruz, Rio de Janeiro, 89 (1): 129.

Arzua, M. \& D.M. Barros-Battesti. 1999. Parasitism of Ixodes (Multidentatus) auritulus Neumann (Acari: Ixodidae) on birds from the city of Curitiba, State of Paraná, Southern Brazil. Memórias do Instituto Oswaldo Cruz, Rio de Janeiro, 94 (5): 597-603.

Arzua, M.; M.A.N. Silva; K.M. Famadas; L. Beati \& D.M. BarrosBATTESTI. 2003. Amblyomma aureolatum and Ixodes auritulus (Acari: Ixodidae) on birds in southern Brazil, with notes on their ecology. Experimental and Applied Acarology, Netherlands, 31: 283-296.

Barros, D.M. \& D. Baggio. 1992. Ectoparasites Ixodida Leach, 1817 on wild mammals in the State of Paraná, Brazil. Memórias do Instituto Oswaldo Cruz, Rio de Janeiro, 87 (2): 291-296.

Barros-Battesti, D.M. \& I. Knysak. 1999. Catalogue of the Brazilian Ixodes (Acari: Ixodidae) material in the mite collection of the Instituto Butantan, São Paulo, Brazil. Papéis Avulsos de Zoologia, São Paulo, 41 (3): 49-57.

Barros-Battesti, D.M.; M. Arzua; M. Pichorim \& J.E. Keirans. 2003. Ixodes (Multidentatus) paranaensis sp. nov. (Acari: Ixodidae) a parasite of Streptoprocne biscutata (Sclater 1865) (Apodidae) birds in Brazil. Memórias do Instituto Oswaldo Cruz, Rio de Janeiro, 98 (1): 93-102.

Beldomenico, P.M.; C.J. Baldi; L.R. Antoniazzi; G.M. Orduna; M. Mastropaolo; A.C. Macedo; M.F. Ruiz; V.M. Orcellet; J.L. PeRalta; J.M. Venzal; A.J. Mangold \& A.A. Guglielmone. 2003. Ixodid Ticks (Acari: Ixodidae) present at Parque Nacional El Rey, Argentina. Neotropical Entomology, Londrina, 32 (2): 273-277.

Evans, D.E.; J.R. Martins \& A.A. Guglielmone. 2000. A review of the ticks (Acari: Ixodida) of Brazil, their hosts and geographic distribution - 1. The State of Rio Grande do Sul, Southern Brazil. Memórias do Instituto Oswaldo Cruz, Rio de Janeiro, 95 (4): 453-470.

Falce, H.C.; C.H.W. Flechtmann \& B.C. Fernandes. 1983. Ixodidae (Acari) on horses, mules and asses in the Paraná, Brazil. Revista da Faculdade de Medicina Veterinária e Zootecnia, São Paulo, 20 (2): 103-106.

Fairchild, G.B.; G.M. Kohls \& V.J. Tipton. 1966. The ticks of Panama (Acarina: Ixodoidea), p.167-219. In: R.L Wenzel \&

Revista Brasileira de Zoologia 22 (3): 623-632, setembro 2005 
V.J. Tipton (Eds). Ectoparasites of Panama. Chicago, Field Museum of Natural History, 825p.

Fonseca, G.A.B.; G. Herrmann; Y. L. R. Leite; R. A. Mittermeier; A. B. Rylands \& J. PATton. 1996. Lista anotada dos mamíferos do Brasil. Conservation International, Washington, 4: 1-38.

Guerra, R.M.S.N.C. \& N.M. Serra-Freire. 1999. Amblyomma oblongoguttatum Koch, 1844 (Acari: Ixodida) in Carollia perspicillata (L.) (Chiroptera: Phyllostomidae): report and ecological reflexion. Entomologia y Vectores, Rio de Janeiro, 6 (1): 63-73.

Guglielmone, A.A.; A.J. Mangold \& C.R. Aufranc. 1992. Haemaphysalis juxtakochi, Ixodes pararicinus (Ixodidae) and Otobius megnini (Argasidae) in relation to the phytogeography of Argentina. Annales de Parasitologie Humaine et Comparee, Paris, 67: 91-93.

Guglielmone, A.A.; A. Estrada-Peña; A.J. Mangold; D.M. BarrosBattesti; M.B. Labruna; J.R. Martins; J.M. Venzal; M. Arzua \& J.E. KeIrans. 2003. Amblyomma aureolatum (Pallas, 1772) and Amblyomma ovale Koch, 1844 (Acari: Ixodidae): hosts, distribution and 16S rDNA sequences. Veterinary Parasitology, Amsterdam, 113 (3-4): 273-288.

Guimarães, L.R. 1945. Sobre alguns ectoparasitos de aves e mamíferos do litoral paranaense. Arquivos do Museu Paranaense, Curitiba, 4: 179-90.

Guimarães, J.H.; E.C. Tucci \& D.M. Barros-Battesti. 2001. Ectoparasitos de Importância Veterinária. São Paulo, Plêiade, 213p.

Jones, E.K.; C.M. Clifford; J.E. Keirans \& G.M. Kohls. 1972. The ticks of Venezuela (Acarina: Ixodoidea) with a key to the species of Amblyomma in the western Hemisfere. Provo, Brigham Young University Science Bulletin, $4^{\text {th }}$ ed., $38 \mathrm{p}$.

Labruna, M.B.; V.S.F. Homem; M.B. Heinemann \& J.S.F. Neto. 2000. Ticks (Acari: Ixodidae) associated with rural dogs in Uruará, Eastern Amazon, Brazil. Journal of Medical Entomology, Lanham, 37 (5): 774-776.

Labruna, M.B.; S.L.P. Souza; J.S. Guimarães; R.C. Pacheco; A. Pinter \& S.M. Gennari. 2001. Prevalência de carrapatos em cães de áreas rurais da região norte do Estado do Paraná. Arquivo Brasileiro de Medicina Veterinária e Zootecnia, Belo Horizonte, 53 (5): 553-556.

Labruna, M.B.; M.J.N. Silva; M.F. Oliveira; D.M. Barros-Battesti \& J.E. KeIRANS. 2003. New records and laboratory-rearing data for Ixodes schulzei (Acari: Ixodidae) in Brazil. Journal of Medical Entomology, Lanham, 40 (1): 116-118.

Marques, S.; D.M. Barros-Battesti; J.L.H. Faccini \& V.C. Onofrio. 2002. Brazilian distribution of Amblyomma varium Koch, 1844 (Acari: Ixodidae), a common parasite of sloths (Mammalia: Xenarthra). Memórias do Instituto Oswaldo Cruz, Rio de Janeiro, 97 (8): 1141-1146.

Mendez-Arocha, M. \& I. Ortiz. 1958. Revision de las garrapatas venezolanas del género Ixodes Latreille, 1725 y estudio de un nuevo Amblyomma (Acarina: Ixodidae). Memoria Sociedad de Ciencias Naturales "La Salle", Caracas, 51 (18): 196-208.

Ibama (Ministério do Meio Ambiente). 2003. Lista nacional das espécies de aves brasileiras. Disponível em: www.ibama. gov.br/cemave/lista/aves-br. Acesso em 10 de setembro de 2004.

Ribeiro, S.S. 1966/1967. Ixodides encontrados no Paraná, distribuição geográfica das espécies brasileiras. Anais da Faculdade de Medicina da Universidade Federal do Paraná, Curitiba, 9-10 (1-2): 7-51.

Ribeiro, S.S. 1970/1971. Ixodídeos encontrados no cão doméstico no Estado do Paraná. Anais da Faculdade de Medicina da Universidade Federal do Paraná, Curitiba, 13-14 (1-2): 61-67.

Rojas, R.; M.A. Marini \& M.T.Z. Coutinho. 1999. Wild birds as hosts of Amblyomma cajennense (Fabricius, 1787) (Acari: Ixodidae). Memórias do Instituto Oswaldo Cruz, Rio de Janeiro, 94 (3): 315-322.

Sinkoc, A.L.; J.G.W. Brum; W. Moraes \& P. Crawshaw. 1998. Ixodinae parasitos de animais silvestres na região do Iguaçu, Brasil e Argentina. Arquivos do Instituto Biológico, São Paulo, 65 (1): 29-33.

Received in 19.X.2004; accepted in 01.VIII.2005. 